\title{
Coleman automorphisms of the normalizers of finite $p$-subgroups
}

\author{
Lv Ruizhen $^{a^{*}}$, Hai Jinke ${ }^{b}$ and Jin Chengcheng ${ }^{c}$ \\ School of Mathematics and Statistics, Qingdao University, Shandong 266071, P.R.China

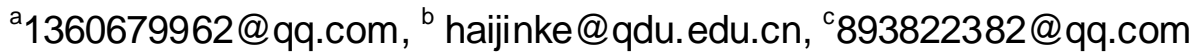

Keywords: Coleman automorphism; normalizer; minimal normal subgroup

\begin{abstract}
Let $G$ be a finite group and $P$ be a $p$-subgroup of $G$, where $p$ is arbitrary prime dividing of $|G|$. If $P$ is abnormal in $G$ or the normalizer of $P$ in $G$ is a $p$-group, then every Coleman automorphism of $G$ is an inner automorphism. Interest in such automorphisms arose from the study of the normalizer problem for integral group rings.
\end{abstract}

\section{Introduction}

Let $G$ be a finite group and let $\sigma$ be an automorphism of $G$. Recall that $\sigma$ is said to be a Coleman automorphism if the restriction of $\sigma$ to any Sylow subgroup of $G$ equals the restriction of some inner automorphisms of $G$. These automorphisms form a characteristic subgroup $A u t_{C o l}(G)$ of automorphisms of $G$. It is clear that $\operatorname{Inn}(G)$ is a normal subgroup of $A u t_{C o l}(G)$. Set $\mathrm{Out}_{C o l}(G)$ $=A u t_{C o l}(G) / \operatorname{Inn}(G)$, which is said to be Coleman outer automorphism of $G$. E.C.Dade has studied lot's of Coleman automorphism about finite group in [2], and he proved that $\mathrm{Out}_{C o l}(G)$ is a nilpotent group. Recently, M. Hertweck and W. Kimmerle proved that $\mathrm{Out}_{\mathrm{Col}}(G)$ is a commute group in [1], and they got some sufficient conditions of Coleman outer automor-phisms group of $G$ is a $p^{\prime}$-group. Moreover, if $G$ is a nilpotent group, then every Coleman automorphism of $G$ is an inner automorphism, i.e., Out $_{C o l}(G)=1$. More generally, if $G$ is a quasinilpotent group, M. Hertweck and W. Kimmerle also proved that every Coleman automorphism of $G$ is an inner automorphism.

The aim of the present paper is to investigate the influence of the normalizers of finite $p$ subgroups on Coleman automorphism. We show some sufficient conditions for $\operatorname{Out}_{C o l}(G)=1$. We can prove the following main result (Theorem 3.1).

Theorem A. Let $G$ be a finite group and $p$ be arbitrary prime dividing of $|G|$. If every $p$ subgroup of $G$ is abnormal in $G$, then every Coleman automorphism of $G$ is an inner automorphism, i.e., $\mathrm{Out}_{\mathrm{Col}}(G)=1$.

Moreover, we have the following result (Theorem 3.3).

Theorem B. Let $G$ be a finite group and $p$ be arbitrary prime dividing of $|G|$. If every normalizer of $p$-subgroups of $G$ is a $p$-group, then every Coleman automorphism of $G$ is an inner automorphism, i.e., $O u t_{C o l}(G)=1$.

\section{Notation and preliminaries}

In this section, we first fix some nation and then record some lemmas that will be used in the sequel. Let $G$ be a finite group. We write $\operatorname{conj}(x)$ for the inner automorphism of $G$ induced by $x$ via conjugacy, i.e., $y^{\operatorname{conj}(x)}=y^{x}$ for any $y \in G$. Denote by $\pi(G)$ the set of all primes dividing the order of $G$. Let $H \leq G, \sigma \in \operatorname{Aut}(G)$. We write $\left.\sigma\right|_{H}$ for the restriction of $\sigma$ to $H$. Let $p \in \pi(G)$. Denote by $P \in \operatorname{Syl}_{p}(G)$ the set of all Sylow $p$-subgroup of $G$. Respectively, we denote an unique 
largest normal $p$-subgroup of $G$ by $O_{p}(G)$. Other notation used will be mostly standard, refer to [4].

In this paper, we present some results which will be used in the proof of the main theorem.

Definition 2.1. Let $G$ be a group, $H \leq G . H$ is said to be abnormal in $G$ if, for every $g \in G$, $g \in<H, H^{g}>$.

Definition 2.2. Let $\sigma \in \operatorname{Aut}(G), p \in \pi(G) . \sigma$ is said to be $p$-center automorphism of $G$ if, there is $P \in S y l_{p}(G)$, such that $\left.\sigma\right|_{P}=\left.i d\right|_{P}$.

Lemma 2.3. Let $G$ be a simple group. Then every Coleman automorphism of $G$ is an inner automorphism, i.e., Out $_{\text {Col }}(G)=1$.

Proof. If $G$ is an abelian simple group, then $G$ is a quasinilpotent group, there is nothing to prove. Let $G$ be a non-abelian simple group. It is clear that no chief factor of $G$ is isomorphic to $C_{p}$ for each $p \in \pi(G)$, and $Z\left(F^{*}(G)\right)=1$. It follows from [1, Theorem 21] that $O u t_{C o l}(G)$ is a $p^{\prime}$ group. By choice of $p$, then every Coleman automorphism of $G$ is an inner automorphism, i.e., Out $_{C o l}(G)=1$.

Lemma 2.4[1, Theorem 14]. Let $G$ be a simple group. Then there is a prime $p \in \pi(G)$ such that $p$-central automorphisms of $G$ are inner automorphisms.

Lemma 2.5[4, Theorem 8.10]. let $G$ be a non-trivial finite group. Then $G$ is characteristically simple if and only if $G$ is a direct product of finitely many isomorphic copies of a simple group.

Lemma 2.6[4, Lemma5.13]. Let $L$ be a finite normal subgroup of $G$, and let $P \in S y l_{p}(G)$. Then $G=N_{G}(P) L$.

Lemma 2.7 [3, Lemma 2]. Let $p$ be a prime, and $\sigma$ be a $p$-power order automorphism of a finite group $G$. Assume further that there is a normal subgroup $K$ of $G$, such that $\sigma$ fixes all elements of $K$, and that $\sigma$ induces the identity on the quotient group $G / K$. Then $\sigma$ induces the identity on $G / O_{p}(Z(K))$.

Definition 2.8. A group $K$ is said to be complete if $Z(K)=1$ and $\operatorname{Aut}(K)=\operatorname{Inn}(K)$.

Lemma 2.9. $S_{n}$ is complete for every integer $n \geq 3$ with $n \neq 6$.

For a proof of this result, see [8].

\section{Proof of Main Theorems}

In this paper, we present proofs for Theorems A and B. For convenience, we record Theorem A here as

Theorem 3.1. Let $G$ be a finite group and $p$ be an arbitrary prime of $\pi(G)$. If every $p$ subgroup of $G$ is abnormal in $G$, then every Coleman automorphism of $G$ is an inner automorphism, i.e., Out $_{C o l}(G)=1$.

Proof. Let $P$ be a $p$-subgroup of $G$ and $P$ be abnormal in $G$. We firstly shows that $N_{G}(P)=P$. In fact, it is clear that $P \leq N_{G}(P)$. Next, let $x \in N_{G}(P)$, then $P^{x}=P$. Since $P$ is abnormal in $G$, it follows that $x \in<P, P^{x}>$, which implies that $x \in P$. Hence $N_{G}(P)=P$. Let $L$ be a minimal normal subgroup of $G$. Since $p$ is an arbitrary prime divisor of $|G|$, we may assume that $P^{\prime}$ is a non-trivial Sylow $p$-subgroup of $L$. Note that $P^{\prime}$ is also abnormal in $G$, it follows that $N_{G}\left(P^{\prime}\right)=P^{\prime}$. By Lemma 2.6, we have $G=N_{G}\left(P^{\prime}\right) L$, which implies that $G=L$, that is, $G$ is minimal. Therefore $G$ is a simple group. It follows from Lemma 2.3 that every Coleman automorphism of $G$ is an inner automorphism, i.e., $O u t_{C o l}(G)=1$. 
Corollary 3.2. Let $P$ be an $p$-subgroup of a finite group $G$ and $p$ be an arbitrary prime of $\pi(G)$. Whenever $g \in G, H \leq G$, and $P \leq H \cap H^{g}$, which implies that $g \in H$. Then every Coleman automorphism of $G$ is an inner automorphism, i.e., $O u t_{C o l}(G)=1$.

Proof. Let $g \in G$, write $H=<P, P^{g}>$, then $H \leq G$, and $P, P^{g} \leq H$. It follows that $P \leq H^{g^{-1}}$, which implies that $P \leq H \cap H^{g^{-1}}$. Note that $g^{-1} \in H$, which implies that $g \in<P, P^{g}>$. Thus $P$ is abnormal in $G$. It follows from Theorem 3.1 that every Coleman automorphism of $G$ is an inner automorphism, i.e., $\mathrm{Out}_{\mathrm{Col}}(G)=1$.

Moreover, we have the following result (Theorem B).

Theorem 3.3. Let $P$ be a $p$-subgroup of a finite group $G$ and $p$ be an arbitrary prime of $\pi(G)$. If $N_{G}(P)$ is $p$-group, then every Coleman automorphism of $G$ is an inner automorphism, i.e., Out $_{C o l}(G)=1$.

Proof. Let $\sigma$ be an Coleman automorphism of $G$ of $p$-power order, we have to show that $\sigma \in \operatorname{Inn}(G)$. Since $G$ is a finite group, then there are minimal normal subgroups of $G$.

Suppose that $G$ has a non-trivial soluble minimal normal subgroup $M$. Then there exists a $q \in \pi(G)$ such that $O_{q}(M) \neq 1$. Since $O_{q}(M) \triangleleft G$, which implies that $N_{G}\left(O_{q}(M)\right)=G$. By hypothesis, $N_{G}\left(O_{q}(M)\right)$ is a $q$-group, i.e., $G$ is a $q$-group. It follows from definition of Coleman automorphism that $\sigma \in \operatorname{Inn}(G)$.

Now, let $L$ be a insoluble minimal normal subgroup of $G$. By Lemma 2.5, we may assume that $L=S_{1} \times S_{2} \times \cdots \times S_{t}$ with isomorphic simple group $S_{i}(i=1,2, \cdots t)$. Let $P \in S y l_{p}(L)$. By Sylow's theorem and note that $N_{G}(P)$ is a $p$-group, then there exists a Sylow $p$-subgroup $D$ of $G$ such that $N_{G}(P) \leq D$, it follows from definition of Coleman automorphism that there exist some $x \in G$, such that $\left.\sigma\right|_{D}=\left.\operatorname{conj}(x)\right|_{D}$, thus $\left.\sigma \operatorname{conj}\left(x^{-1}\right)\right|_{D}=\left.i d\right|_{D}$, we write $\beta=\sigma \operatorname{conj}\left(x^{-1}\right)$, so $\left.\beta\right|_{D}=\left.i d\right|_{D}$, we obtain that $\left.\beta\right|_{N_{G}(P)}=\left.i d\right|_{N_{G}(P)}$ and $\left.\beta\right|_{P}=\left.i d\right|_{P}$. Since $S_{i} \cong S_{j}$, where $i, j=1,2, \cdots t$, without loss of generality, we may assume that $S_{i}^{\beta}=S_{i}$, thus $\beta \in A u t\left(S_{i}\right)(i=1,2, \cdots t)$. By Lemma 2.6, we have $G=N_{G}(P) L$, which implies that

$$
\left.\beta\right|_{G / L}=\left.i d\right|_{G / L}
$$

Let $P_{i}$ be Sylow $p$-subgroup of $S_{i}$. Without loss of generality, we may assume that $P_{i} \leq P$, hence $\left.\beta\right|_{P_{i}}=\left.i d\right|_{P_{i}}(i=1,2, \cdots t)$. Since $S_{i}$ is a simple group, it follows from Lemma 2.4 that $\beta$ is an inner automorphism of $S_{i}$, i.e., $\left.\beta\right|_{S_{i}} \in \operatorname{Inn}\left(S_{i}\right)(i=1,2, \cdots t)$. Note that $\operatorname{Inn}\left(S_{1} \times S_{2} \times \cdots \times S_{t}\right)=\operatorname{Inn}\left(S_{1}\right)$ $\times \operatorname{Inn}\left(S_{2}\right) \times \cdots \times \operatorname{Inn}\left(S_{t}\right)$ Therefore $\left.\beta\right|_{S_{1} \times S_{2} \times \cdots \times S_{t}} \in \operatorname{Inn}\left(S_{1} \times S_{2} \times \cdots \times S_{t}\right)$, i.e., $\left.\beta\right|_{L} \in \operatorname{Inn}(L)$. Then there are some $y \in L \operatorname{such}$ that $\left.\left.\beta\right|_{L} \in \operatorname{conj}(y)\right|_{L}$, so $\left.\beta \operatorname{conj}\left(y^{-1}\right)\right|_{L}=\left.i d\right|_{L}$, we write $\theta=\beta \operatorname{conj}\left(y^{-1}\right)$, then

$$
\left.\theta\right|_{L}=\left.i d\right|_{L}
$$

By (1) and (2),

$$
\left.\theta\right|_{G / L}=\left.i d\right|_{G / L}
$$

By (2), (3) and Lemma 2.7, we have

$$
\left.\theta\right|_{G / O_{p} Z(L)}=\left.i d\right|_{G / O_{p} Z(L)} .
$$


Since $L$ is an insoluble minimal normal subgroup of $G$, which implies that $Z(L)=1$, so $O_{p}(Z(L))=1$, by (4), we have $\left.\theta\right|_{G}=\left.i d\right|_{G}$. Hence we obtain that $\theta \in \operatorname{Inn}(G)$. Implying further that $\sigma \in \operatorname{Inn}(G)$, we are done.

As immediate consequences of Theorem 3.1 and Theorem 3.3, we have the following result:

Corollary 3.4. Let $P$ be an arbitrary Sylow $p$-subgroup of a finite group $G$ and $p$ be an arbitrary prime of $\pi(G)$. If $P$ is abnormal in $G$, and $N_{G}\left(P_{1}\right) \leq N_{G}(P)$, where $P_{1} \leq P$. Then every Coleman automorphism of $G$ is an inner automorphism, i.e., $O u t_{C o l}(G)=1$.

Proof. Since $P$ is abnormal in $G$, it follows from Theorem 3.1 that $N_{G}(P)=P$. Note that, if $P_{1} \leq P$, then $N_{G}\left(P_{1}\right) \leq N_{G}(P)$. By choice of $p$, normalizer of every $p$-subgroup of $G$ is $p$-group. By Theorem 3.3, then every Coleman automorphism of $G$ is an inner automorphism, i.e., Out $_{\text {Col }}(G)=1$.

More generally, we have the following result .

Examples 3.5. Let $S_{n}$ be a symmetric group of degree $n$, where $n$ is a positive integer. Then every Coleman automorphism of $S_{n}$ is an inner automorphism, i.e., $O u t_{C o l}\left(S_{n}\right)=1$.

Proof. Let $p \in \pi\left(S_{n}\right)$ and let $\sigma$ be an Coleman automorphism of $S_{n}$ of $p$-power order. We have to show that $\sigma$ is an inner automorphism of $S_{n}$.

This is clear for $n \leq 2$. If $n \geq 3$ with $n \neq 6$, we have $S_{n}$ is complete group, it follows from Definition 2.8 that, $\operatorname{Aut}\left(S_{n}\right)=\operatorname{Inn}\left(S_{n}\right)$. So $\sigma \in \operatorname{Inn}\left(S_{n}\right)$. It remains to consider the case $S_{6}$. Since $\left|\operatorname{Aut}\left(S_{6}\right) / \operatorname{Inn}\left(S_{6}\right)\right|=2$, we may assume that $\sigma$ is a Coleman automorphism of 2-power order. Let $P \in \operatorname{Syl}_{2}\left(S_{6}\right)$ with $(12) \in P$. Then, by the definition of Coleman automorphism, there exists some $x \in S_{6}$, such that $\left.\sigma\right|_{P}=\left.\operatorname{conj}(x)\right|_{P}$. In particular, $\sigma$ sends the transposition (12) of $S_{6}$ to some conjugate of it. But it is known that any outer automorphism of $S_{6}$ maps the transposition (12) to a permutation of type $(2,2,2)$. Which implies that $\sigma \in \operatorname{Inn}\left(S_{6}\right)$, we are done.

\section{References}

[1] M. Hertweck, W. Kimmerle, Coleman automorphisms of finite groups. Math Z., 242 (2002) 203-215.

[2] E. C. Dade, Sylow-centralizing sections of outer automorphism groups of finite groups are nilpotent. Math. Z., 141 (1975) 57-76.

[3] M. Hertweck. Class-preserving automorphisms of finite groups. J. Algebra, 241 (2001) 1-26.

[4] J. S. Rose. A course on group theory. Cambridge University Press, Cambridge-New YorkMelbourne, 1978.

[5] Zhengxing Li, Jinke Hai, Shuxian Yang. Coleman automorphisms of finite groups with a unique nontrivial normal subgroup. J. Math Research with Applications. 34 (2014) 301-306.

[6] Jinke Hai, Yulei Wang. Some sufficient conditions for the Coleman outer automorphism group of a finite group being a $p^{\prime}$-group. J. Math. 28 (2008) 653-658.

[7] H. Kurzweil, B. Stellmacher,The theory of finite groups, an Introduction, Spinger-Verlag, New York.Berlin. Heidelberg, 2004.

[8] A. G. Kurosh. The theory of groups. 2nd English ed., Chelsea 1960. 